NISSUNA UMANA INVESTIGAZIONE SI PUO DIMANDARE VERA SCIENZIA S'ESSA NON PASSA PER LE MATEMATICHE DIMOSTRAZIONI LEONARDO DA VINCI

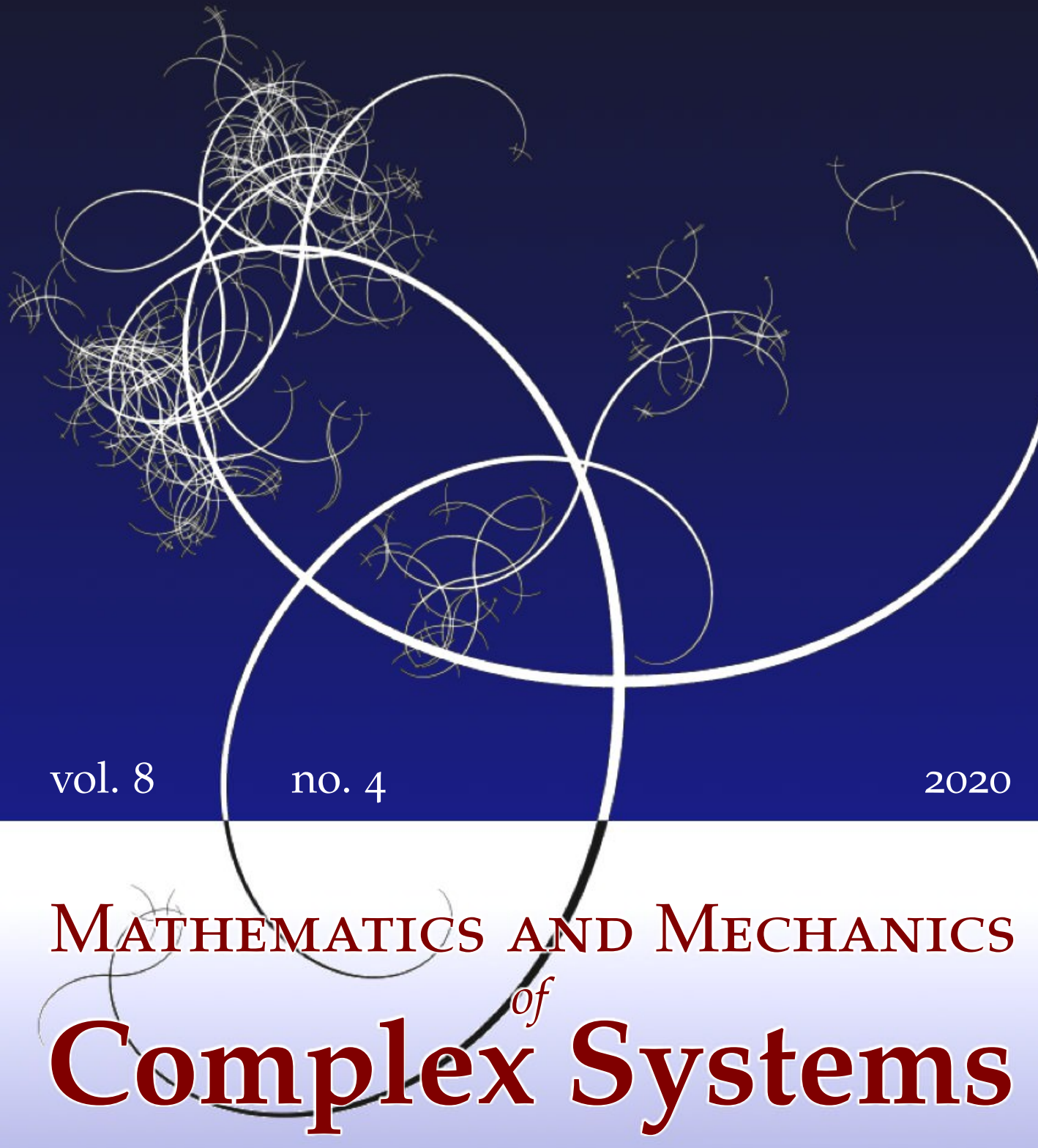

LOC V. TRAN AND JARKKO NIIRANEN

A GEOMETRICALLY NONLINEAR EULER-BERNOULLI BEAM MODEL WITHIN STRAIN GRADIENT ELASTICITY

WITH ISOGEOMETRIC ANALYSIS

AND LATTICE STRUCTURE APPLICATIONS 


\title{
A GEOMETRICALLY NONLINEAR EULER-BERNOULLI BEAM MODEL WITHIN STRAIN GRADIENT ELASTICITY WITH ISOGEOMETRIC ANALYSIS AND LATTICE STRUCTURE APPLICATIONS
}

\author{
LOC V. TRAN AND JARKKO NIIRANEN
}

\begin{abstract}
The nonlinear governing differential equation and variational formulation of the Euler-Bernoulli beam model are formulated within Mindlin's strain gradient elasticity theory of form II by adopting the von Kármán strain assumption. The formulation can retrieve some simplified beam models of generalized elasticity such as the models of simplified strain gradient theory (SSGT), modified strain gradient theory (MSGT), and modified couple stress theory (MCST). Without the presence of nonlinear terms, the resulting linear differential equation is solvable by analytical means, whereas the mathematical complexity of the nonlinear problem is treated with the Newton-Raphson iteration and a conforming isogeometric Galerkin method with $C^{p-1}$-continuous B-spline basis functions of order $p \geq 3$. Through a set of numerical examples, the accuracy and validity of the present theoretical formulation at linear and nonlinear regimes are confirmed. Finally, an application to lattice frame structures illustrates the benefits of the present beam model in saving computational costs, while maintaining high accuracy as compared to standard $2 \mathrm{D}$ finite element simulations.
\end{abstract}

\section{Introduction}

Microbeams are nowadays the key components in micro- and nanoelectromechanical systems (MEMS and NEMS, respectively) which are broadly applicable in designs such as microsensors and -actuators [Hu et al. 2004; Lun et al. 2006; Moghimi Zand and Ahmadian 2009], atomic force microscopes [Chang et al. 2007; Turner and Wiehn 2001], and so on. In these devices, the beam thickness is sized down to the order of microns and submicrons. A number of experimental tests [Fleck et al. 1994; Lam et al. 2003; Stölken and Evans 1998] have demonstrated, however, that the size-dependent behavior of these extremely small-scale

\section{Communicated by Victor A. Eremeyev.}

This work was supported by the Academy of Finland (grant number 304122).

MSC2010: 65M60, 74A60, 74B20, 74K10, 74Q15.

Keywords: strain gradient elasticity, geometric nonlinearity, beam model, isogeometric analysis, lattice structure. 
microstructural systems cannot be predicted and explained by classical continuum mechanics. In fact, experimental measurements have revealed that reducing the beam/rod size, e.g., decreasing the beam/rod thickness or diameter, results in an enhancement of the torsional stiffness of a copper wire [Fleck et al. 1994], a significant increase in the level of plastic hardening of a thin nickel beam [Stölken and Evans 1998], or a remarkable increase in the bending rigidity of an epoxy beam [Lam et al. 2003]. Interestingly, another class of structures sharing the same nature is microarchitectural structures of any scale [dell'Isola et al. 2016; Khakalo et al. 2018; Khakalo and Niiranen 2019]: size-dependent behavior is an inherent property of materials or metamaterials, present when the characteristic length of the material microstructure becomes comparable with the dimensions of the structure itself, such as the thickness of thin structures. This leads to the necessity of nonclassical continuum theories which include material length scale parameters for predicting size effects, in addition to the classical Lamé constants used in the conventional theory of elasticity.

The nonclassical continuum theories can be classified into two branches: "higherorder" theories proposing additional (internal) variables [Cosserat and Cosserat 1909; Eringen 1999; Green and Rivlin 1964] and "higher-grade" theories including higher gradients of the classical variables, displacements, or strains. In the latter, one of the most well known theories is the strain gradient elasticity theory pioneered by Mindlin [1964; Mindlin and Eshel 1968] and other contemporaries. In the restriction of the present work, we focus only on the strain gradient theory of form II in which the second derivatives of strains are involved. It is worth noting that the three-dimensional isotropic version of Mindlin's theory employs five additional material parameters as compared to the classical isotropic elasticity. Over the last fifty years, many versions of Mindlin's original formulation have been proposed [Lam et al. 2003; Aifantis 1992; Yang et al. 2002] in order to introduce fewer additional material parameters. In the framework of strain gradient elasticity theory, Aifantis's proposal [1992] for a nonlocal version of the generalized Hooke's law introduced only one length scale parameter beside the two conventional Lamé parameters. The corresponding variational formulation was introduced by Altan and Aifantis [1997]. In the framework of this simplified strain gradient theory (SSGT), bending and vibration analysis of beam- and platelike structures, in particular, has been accomplished in [Lazopoulos 2004; 2012; Lazopoulos and Lazopoulos 2010; Askes and Aifantis 2009; Niiranen et al. 2019; 2017; Balobanov and Niiranen 2018]. Lam et al. [2003] simplified Mindlin's formulation to the so-called modified strain gradient theory (MSGT) involving three material length scale parameters. By eliminating two of them, Yang et al. [2002] suggested a modified couple stress theory (MCST) with one additional material parameter again. Based on these two theories, many works involving static and 
dynamic investigations of linear Euler-Bernoulli and Timoshenko beams have been published [Park and Gao 2006; Ma et al. 2008; Kong et al. 2009; Wang et al. 2010]. The reviews in [Lurie and Solyaev 2018; Thai et al. 2017] are suggested for a detailed exposition. Regarding more general models incorporating more nonclassical constitutive parameters but still fewer than in the full anisotropic form of Mindlin's theory, we refer to the following recent contributions: an anisotropic form of the so-called weak nonlocality [Lazar and Po 2015], an anisotropic version of Mindlin's form-II thermoelasticity [Khakalo and Niiranen 2020], a simplified version of Mindlin's second strain gradient (third displacement gradient) elasticity [Khakalo and Niiranen 2018], or microarchitecture-specific second displacement gradient formulations; see, e.g., [Boutin et al. 2017; Rickert et al. 2019; dell'Isola et al. 2019a; 2019b; Abdoul-Anziz and Seppecher 2018].

As seen, the studies in [Lazopoulos 2004; 2012; Lazopoulos and Lazopoulos 2010; Askes and Aifantis 2009; Niiranen et al. 2019; 2017; Balobanov and Niiranen 2018; Park and Gao 2006; Ma et al. 2008; Kong et al. 2009; Wang et al. 2010] are restricted to the linear regime of structural analysis. However, the beam structures used in MEMS or NEMS, or microarchitectural structures, can exhibit large deformations in which the stretching becomes dominant, which results in geometrical nonlinearity which, in turn, results in significant changes in the structural response in both statics and dynamics [Hassanpour et al. 2010; Abdel-Rahman et al. 2002]. Therefore, beside the linear investigations listed above, studies on nonlinearities have gotten attention. For instance, Xia et al. [2010] developed a nonlinear Euler-Bernoulli model based on MCST for the analysis of statics, free vibration, and postbuckling. Asgharis et al. [2010; 2012; Kahrobaiyan et al. 2011] studied the same beam problems by using a nonlinear Timoshenko beam model. Lazopoulos et al. formulated the nonlinear bending and buckling problems of beams [Lazopoulos et al. 2014] and shallow shells [Lazopoulos and Lazopoulos 2011]. In addition, Ramezani [2012; 2013] adopted the multiple scales perturbation technique to solve analytically the geometrically nonlinear beam and plate problems based on strain gradient elasticity. As observed in the aforementioned works, the governing differential equations in the framework of strain gradient theory are mathematically complex due to the appearance of many nonlinear terms involving higher-order derivatives of the variables. Generally, analytical approaches can be utilized only in some simple cases of geometries, loadings, and boundary conditions. Therefore, numerical techniques are necessary. Furthermore, the numerical tools must be somewhat special in cases which require higher-order continuity. Dadgar-Rad and Beheshti [2017] proposed a novel two-node microbeam element based on using fifth-order Hermite functions in order to deal with the stringent continuity requirements. By another way, Hughes et al. [2005] proposed an isogeometric analysis (IGA) utilizing the same basis functions as a B-spline or NURBS in describing the 
geometry to construct the finite approximation. Literature on the computational application of IGA is extremely vast, especially for the beam and plate problems [Bauer et al. 2016; Kiendl et al. 2015; Luu et al. 2015; Tran et al. 2013; Thai et al. 2014; Vo and Nanakorn 2020; Greco and Cuomo 2014; Greco et al. 2017; Greco 2020]. One of the most salient features of the IGA shown clearly in Niiranen's works [Niiranen et al. 2019; 2017] is to use a conforming isogeometric $C^{p-1}$ continuous discretization (with order $p \geq 3$ ) to naturally fulfill the required $C^{2}$ continuity requirement without any additional variables. In the present work, we extend this approach to nonlinear deformations.

First, we formulate a nonlinear strain-gradient-elastic beam model based on Mindlin's strain gradient elasticity theory of form II [Mindlin 1964]. The formulation takes into account the von Kármán strain tensor for geometrical nonlinearity. With proper choices of length scale parameters, we retrieve various one-parameter beam models corresponding to SSGT, MSGT, and MCST and a relation between these models.

Second, we adopt isogeometric B-spline basis functions for implementing a conforming $C^{p-1}$-continuous Galerkin method. Then by applying the NewtonRaphson method, the nonlinear beam bending problem is solved iteratively. Through a set of numerical benchmarks, the accuracy and validity of the present theoretical formulations at linear and nonlinear regimes are confirmed.

Third, we demonstrate the advantages of applying the strain gradient elasticity theory for analyzing 2D triangular lattice structures from the linear regime to the regime of the von Kármán-type geometrical nonlinearity. By using a dimension reduction model, we significantly reduce the number of degrees of freedom, which results in essential savings in computational costs, while maintaining a good level of accuracy, as compared to standard 2D finite element simulations. From the theoretical point of view, it is interesting to witness that beam structures having a triangular, stretching-dominated lattice microarchitecture follow the size-dependent generalized beam models, allowing us to extend the results of [Khakalo et al. 2018; Khakalo and Niiranen 2019] concerning the linear regime of the generalized EulerBernoulli and Timoshenko beam models. From the mechanical point of view, it is crucial that these beam models share the kinematical assumption of straight cross-sectional fibers fulfilled by the lattice beams [Khakalo and Niiranen 2019, Appendix C].

This paper is outlined as follows. The next section details the strain gradient elasticity theory for the Euler-Bernoulli beam model adopting the von Kármán strain assumption. In Section 3, we derive a variational formulation of the beam model for which we then write a conforming Galerkin method based on isogeometric analysis. A set of numerical examples is examined in Section 4. Finally, some concluding remarks close the article. 


\section{Continuum models for generalized beams}

2.1. Strain gradient elasticity theory. To capture the size effects of isotropic materials, Mindlin [1964] proposed the strain energy density of a microstructural solid in a general form with an additional higher-order strain gradient tensor $\xi$ beside the infinitesimal strain tensor $\boldsymbol{\varepsilon}$ appearing in the conventional continuum theories:

$$
\begin{aligned}
U(\boldsymbol{\varepsilon}, \boldsymbol{\xi})=\frac{1}{2} \lambda \varepsilon_{i i} \varepsilon_{j j}+\mu \varepsilon_{i j} \varepsilon_{i j} & \\
& +a_{1} \xi_{i i k} \xi_{k j j}+a_{2} \xi_{i j j} \xi_{i k k}+a_{3} \xi_{i i k} \xi_{j j k}+a_{4} \xi_{i j k} \xi_{i j k}+a_{5} \xi_{i j k} \xi_{k j i}
\end{aligned}
$$

where $\lambda$ and $\mu$ are the classical Lamé constants related to Young's modulus and Poisson's ratio as $\lambda=E v /(1-2 v)(1+v)$ and $\mu=E / 2(1+v)$, whereas the $a_{i}$ $(i=1,2, \ldots, 5)$ are nonclassical material parameters. The components of the thirdorder strain gradient tensor $\xi_{i j k}$ are defined according to the type-II formulation of Mindlin's theory as

$$
\xi_{i j k}=\varepsilon_{j k, i}
$$

where the infinitesimal strain tensor $\varepsilon_{i j}$ is written in terms of the displacement components $u_{i}$ according to the Green strain assumption as usual in continuum mechanics:

$$
\varepsilon_{i j}=\frac{1}{2}\left(u_{i, j}+u_{j, i}+u_{k, i} u_{k, j}\right) .
$$

Note that the symbol $(\cdot),{ }_{i}$ denotes the derivative with respect to coordinate $x_{i}$. The constitutive equations for the Cauchy-like stress and double stress are then given by

$$
\begin{aligned}
& \sigma_{i j}=\frac{\partial U}{\partial \varepsilon_{i j}}=\lambda \varepsilon_{k k} \delta_{i j}+\mu \varepsilon_{i j} \\
& \tau_{i j k}=\frac{\partial U}{\partial \xi_{i j k}}=\frac{1}{2} a_{1}\left(\delta_{i j} \xi_{k p p}\right.\left.+2 \delta_{j k} \xi_{p p i}+\delta_{i k} \xi_{j p p}\right)+2 a_{2} \delta_{j k} \xi_{i p p} \\
&+a_{3}\left(\delta_{i j} \xi_{p p k}+\delta_{i k} \xi_{p p j}\right)+2 a_{4} \xi_{i j k}+a_{5}\left(\xi_{j k i}+\xi_{k i j}\right) .
\end{aligned}
$$

By assigning specific values for the additional material parameters $a_{i}$, certain versions of strain gradient theories can be obtained. For instance, Lam et al. [2003] introduced the modified strain gradient theory with three length scale parameters $l_{i}(i=0,1,2)$ which are used to calculate the five independent parameters $a_{i}$ in Mindlin's form II as

$$
\begin{aligned}
& a_{1}=\mu\left(-\frac{4}{15} l_{1}^{2}+l_{2}^{2}\right), \quad a_{2}=\mu\left(l_{0}^{2}-\frac{1}{15} l_{1}^{2}\right), \\
& a_{3}=\mu\left(-\frac{4}{15} l_{1}^{2}-\frac{1}{2} l_{2}^{2}\right), \quad a_{4}=\mu\left(\frac{1}{3} l_{1}^{2}+l_{2}^{2}\right), \quad a_{5}=\mu\left(\frac{2}{3} l_{1}^{2}-l_{2}^{2}\right) .
\end{aligned}
$$

In the special case of $l_{0}=l_{1}=l_{2}=l$, the nonclassical material parameters $a_{i}$ can be written in terms of one additional length scale parameter as

$$
\left\{a_{1}, a_{2}, a_{3}, a_{4}, a_{5}\right\}=\frac{1}{30} \mu l^{2}\{22,13,-23,40,-10\} .
$$


Similarly, Yang et al. [2002] suggested the modified couple stress theory by eliminating $l_{0}$ and $l_{1}$. As a consequence, (6) is rewritten as

$$
a_{1}=-2 a_{2}=-2 a_{3}=a_{4}=-a_{5}=\mu l^{2} .
$$

In the framework of simplified strain gradient theory [Aifantis 1992], only the nonclassical terms related to $a_{2}$ and $a_{4}$ are considered by introducing a material length scale parameter $g$ as

$$
a_{1}=a_{3}=a_{5}=0, \quad a_{2}=\frac{1}{2} g^{2} \lambda, \quad a_{4}=g^{2} \mu .
$$

2.2. Kinematics of Euler-Bernoulli beams. Let us consider a three-dimensional prismatic beam structure with length $L$, thickness $h$, and width $b$. For simplicity, it is assumed that the beam cross-section $A$ is constant. Within the Euler-Bernoulli hypotheses for in-plane bending, the displacement field of an arbitrary point in the beam is defined as

$$
u_{x}(x, y, z)=u(x)-y w^{\prime}(x), \quad u_{y}(x, y, z)=w(x), \quad u_{z}(x, y, z)=0,
$$

where $u$ and $w$ denote the axial extension and transverse displacement of the beam, respectively. Substituting the displacement field of (10) into (3), with such an assumption that the beam can exhibit large deflection but small or moderate rotation, only one nonzero strain component remains active, expressed according to the von Kármán strain formulation as

$$
\varepsilon_{x x}=u_{x}^{\prime}+\frac{1}{2}\left(u_{y}^{\prime}\right)^{2}=\varepsilon_{0}+y \varepsilon_{1} .
$$

Accordingly, two nonzero components of the strain tensor gradient are obtained according to (2):

$$
\begin{aligned}
& \varepsilon_{x x x}=\varepsilon_{x x, x}=\varepsilon_{2}+y \varepsilon_{3}, \\
& \varepsilon_{y x x}=\varepsilon_{x x, y}=\varepsilon_{1},
\end{aligned}
$$

where these variables can be written in a matrix form as

$$
\hat{\boldsymbol{\varepsilon}}=\left[\begin{array}{c}
\varepsilon_{0} \\
\varepsilon_{1} \\
\varepsilon_{2} \\
\varepsilon_{3}
\end{array}\right]=\left[\begin{array}{c}
u^{\prime}+\frac{1}{2}\left(w^{\prime}\right)^{2} \\
-w^{\prime \prime} \\
u^{\prime \prime}+w^{\prime} w^{\prime \prime} \\
-w^{\prime \prime \prime}
\end{array}\right] .
$$

Herein, the prime stands for a derivative with respect to $x$. By following the constitutive equations in (4) and (5), the nonzero Cauchy-like stress and double stress for the beam are given as

$$
\sigma_{x x}=E \varepsilon_{x x}, \quad \tau_{x x x}=2 \sum_{I=1}^{5} a_{I} \varepsilon_{x x x}=\alpha_{1} \varepsilon_{x x x}, \quad \tau_{y x x}=2\left(a_{2}+a_{4}\right) \varepsilon_{y x x}=\alpha_{2} \varepsilon_{y x x} .
$$




\section{Variational formulation and Galerkin-type isogeometric analysis}

3.1. Variational formulation and boundary conditions. The virtual strain energy in the Euler-Bernoulli beam model based on the strain gradient theory has the form

$$
\begin{aligned}
\delta U & =\int_{V}\left(\sigma_{x x} \delta \varepsilon_{x x}+\tau_{x x x} \delta \varepsilon_{x x x}+\tau_{y x x} \delta \varepsilon_{y x x}\right) d V \\
& =\int_{0}^{L} \int_{A}\left(\sigma_{x x}\left(\delta \varepsilon_{0}+y \delta \varepsilon_{1}\right)+\tau_{x x x}\left(\delta \varepsilon_{2}+y \delta \varepsilon_{3}\right)+\tau_{y x x} \delta \varepsilon_{1}\right) d A d x \\
& =\int_{0}^{L}\left(N \delta \varepsilon_{0}+(M+Q) \delta \varepsilon_{1}+R \delta \varepsilon_{2}+P \delta \varepsilon_{3}\right) d x=\int_{0}^{L} \delta \hat{\boldsymbol{\varepsilon}}^{T} \hat{\boldsymbol{\sigma}} d x,
\end{aligned}
$$

where the classical and nonclassical stress resultants are defined as

$$
\begin{aligned}
& N=\int_{A} \sigma_{x x} d A=E A\left[u^{\prime}+\frac{1}{2}\left(w^{\prime}\right)^{2}\right], \quad M=\int_{A} y \sigma_{x x} d A=-E I w^{\prime \prime}, \\
& R=\int_{A} \tau_{x x x} d A=\alpha_{1} A\left(u^{\prime \prime}+w^{\prime} w^{\prime \prime}\right), \quad P=\int_{A} y \tau_{x x x} d A=-\alpha_{1} I w^{\prime \prime \prime}, \\
& Q=\int_{A} \tau_{y x x} d A=-\alpha_{2} A w^{\prime \prime} .
\end{aligned}
$$

We note that $M$ and $N$ represent the classical stress resultants and $N$ includes a nonlinear strain term as usual, whereas $P, Q$, and $R$ are characteristic for the generalized beam models as follows. $P$ is a parameter-dependent higher-order bending term responsible for possible boundary layers depending on boundary conditions [Niiranen et al. 2019]. $Q$ brings a size dependency to the model, which can be revealed as follows [Niiranen et al. 2019]: when $Q$, proportional to $\alpha_{2} A$, which in turn is proportional to $\alpha_{2} h^{2}$, is combined with $M$, proportional to $E I$, being in turn proportional to $E h^{4}$, one obtains a bending term proportional to $E I\left(1+\alpha_{2} / h^{2}\right)$. The first term of $R$ can be identified as a boundary layer term related to the corresponding bar problem [Niiranen et al. 2016], whereas the second term is a nonclassical term having a link to the nonlinear part of strain. In fact, when the second term of $R$ is combined with $M$ and $Q$, it can be interpreted as a stiffening nonlinear term (actually, equal to $-w^{\prime} Q$ by assuming that $\alpha_{1}=\alpha_{2}$ ).

Equation (16) can be rewritten in a compact form as

$$
\hat{\boldsymbol{\sigma}}=[N, M+Q, R, P]^{T}=\boldsymbol{D} \hat{\boldsymbol{\varepsilon}}
$$

in which $\boldsymbol{D}=\operatorname{diag}\left(E A, E I+\alpha_{2} A, \alpha_{1} A, \alpha_{1} I\right)$ forms a diagonal constitutive matrix.

The virtual work done by the external forces $f_{x}$ and $f_{y}$ can be written as

$$
\delta W=\int_{0}^{L}\left(f_{x} \delta u+f_{y} \delta w\right) d x
$$


and finally the principle of virtual work is expressed in the form

$$
\begin{aligned}
0 & =\delta \Pi=-\delta U+\delta W \\
& =-\int_{0}^{L}\left(N \delta \varepsilon_{0}+(M+Q) \delta \varepsilon_{1}+R \delta \varepsilon_{2}+P \delta \varepsilon_{3}\right) d x+\int_{0}^{L}\left(f_{x} \delta u+f_{y} \delta w\right) d x .
\end{aligned}
$$

Let us next integrate by parts (19) until getting terms which contain the virtual displacements $\delta u$ and $\delta w$ as common factors. Thereafter, the strong form as a pair of governing equations is expressed as

$$
\begin{aligned}
& \text { ( } \delta u) \quad-N^{\prime}+R^{\prime \prime}=f_{x} \text {, } \\
& (\delta w)-\left(N w^{\prime}\right)^{\prime}-(M+Q)^{\prime \prime}+\left(R^{\prime} w^{\prime}\right)^{\prime}+P^{\prime \prime \prime}=f_{y} .
\end{aligned}
$$

Additionally, the corresponding boundary conditions are obtained via the following essential (left) or natural (right) conditions:

$$
\begin{array}{rlrlrl}
u & =\bar{u} & \text { or } & N-R^{\prime} & =\bar{N}, \\
u^{\prime} & =\bar{\alpha} & \text { or } & R & =\bar{R}, \\
w & =\bar{w} & \text { or } & & \left(N-R^{\prime}\right) w^{\prime}+(M+Q)^{\prime}-P^{\prime \prime} & =\bar{V}, \\
w^{\prime} & =\bar{\beta} & \text { or } & -(M+Q)+R w^{\prime}+P^{\prime} & =\bar{M}, \\
w^{\prime \prime} & =\bar{\kappa} & \text { or } & P & =\bar{P} .
\end{array}
$$

The overlined symbols above denote prescribed boundary values, as usual.

By substituting the definitions of the stress resultants in (16) into (20), the governing equation can be rewritten in terms of displacements as

$$
\begin{array}{rl}
-E & A\left[u^{\prime \prime}+w^{\prime} w^{\prime \prime}\right]+\alpha_{1} A\left(u^{(4)}+3 w^{\prime \prime} w^{\prime \prime \prime}+w^{\prime} w^{(4)}\right)=f_{x}, \\
-E A\left[u^{\prime} w^{\prime}+\frac{1}{2} w^{\prime 3}\right]^{\prime} & +\left(E I+\alpha_{2} A\right) w^{(4)} \\
& +\alpha_{1} A\left[\left(u^{\prime \prime \prime} w^{\prime}+w^{\prime 2} w^{\prime \prime \prime}+w^{\prime} w^{\prime \prime 2}\right)\right]^{\prime}-\alpha_{1} I w^{(6)}=f_{y} .
\end{array}
$$

In particular, by eliminating the nonlinear terms in (22) we can obtain the equilibrium equations corresponding to the linear form of the strain gradient EulerBernoulli beam model as a pair of decoupled stretching and bending equations [Niiranen et al. 2019]:

$$
\begin{aligned}
-E A u^{\prime \prime}+\alpha_{1} A u^{(4)} & =f_{x}, \\
\left(E I+\alpha_{2} A\right) w^{(4)}-\alpha_{1} I w^{(6)} & =f_{y}
\end{aligned}
$$


with the respective boundary conditions

$$
\begin{array}{rlrlrl}
u & =\bar{u} & \text { or } & E A u^{\prime}-\alpha_{1} A u^{\prime \prime \prime} & =\bar{N}, \\
u^{\prime} & =\bar{\alpha} & \text { or } & \alpha_{1} A u^{\prime \prime} & =\bar{R}, \\
w & =\bar{w} & \text { or } & & -\left(E I+\alpha_{2} A\right) w^{\prime \prime \prime}+\alpha_{1} I w^{(5)} & =\bar{V}, \\
w^{\prime} & =\bar{\beta} & \text { or } & & \left(E I+\alpha_{2} A\right) w^{\prime \prime}-\alpha_{1} I w^{(4)} & =\bar{M}, \\
w^{\prime \prime} & =\bar{\kappa} & \text { or } & \alpha_{1} I w^{\prime \prime \prime} & =\bar{P} .
\end{array}
$$

Regarding (23) and (24), notice that the axial and transverse displacements are prescribed independently.

3.2. Finite element equations. Solving the nonlinear equation (22) even in the simplest cases is a nontrivial task. Therefore, we prefer solving the problem via a weak form equation based on the discrete formulation of (19) by using the isogeometric finite element method [Hughes et al. 2005]. With an open knot vector $\boldsymbol{\Xi}=\left\{\varsigma_{1}, \varsigma_{2}, \ldots, \varsigma_{m+p+1}\right\}$, which is a nondecreasing sequence of parameter values $\varsigma_{i} \in \mathbb{R}^{+}(i=1,2, \ldots, m+p)$ with $m$ denoting the number of basis functions, the univariate B-spline basis functions $\phi_{i}^{p}(\varsigma)$ are defined recursively by using the Coxde Boor algorithm [Piegl and Tiller 1997]:

$$
\begin{aligned}
\phi_{i}^{p}(\varsigma) & =\frac{\varsigma-\varsigma_{i}}{\varsigma_{i+p}-\varsigma_{i}} \phi_{i}^{p-1}(\varsigma)+\frac{\varsigma_{i+p+1}-\varsigma}{\varsigma_{i+p+1}-\varsigma_{i+1}} \phi_{i+1}^{p-1}(\varsigma) \quad \text { if } p \geq 1, \\
\phi_{i}^{0}(\varsigma) & = \begin{cases}1 & \text { if } \varsigma_{i}<\varsigma<\varsigma_{i+1}, \\
0 & \text { otherwise. }\end{cases}
\end{aligned}
$$

Similar to the traditional finite element method, isogeometric analysis invokes the isoparametric concept in which the displacements are approximated by a linear combination of the basis functions and the unknown degrees of freedom in the form

$$
\boldsymbol{u}^{h}=\sum_{I=1} \phi_{I}(\varsigma) d_{I}
$$

where $\boldsymbol{d}_{I}=\left[u_{I}, w_{I}\right]^{T}$ denotes the degrees of freedom associated to control point $I$.

According to the approximate displacement in (26), the variation of the strain vectors denoted by $\delta \hat{\boldsymbol{\varepsilon}}$ can be computed as

$$
\delta \hat{\boldsymbol{\varepsilon}}=\sum_{I} \boldsymbol{B}_{I} \delta \boldsymbol{d}_{I}
$$

where the generalized strain matrix is defined as

$$
\boldsymbol{B}_{I}=\left[\begin{array}{cccc}
\phi_{I}^{\prime} & 0 & \phi^{\prime \prime} & 0 \\
w^{\prime} \phi_{I}^{\prime} & -\phi_{I}^{\prime \prime} & w^{\prime} \phi_{I}^{\prime \prime}+w^{\prime \prime} \phi_{I}^{\prime} & -\phi_{I}^{\prime \prime \prime}
\end{array}\right]^{T} .
$$


As seen, the third derivative of the basic function is required in (28). Therefore, at least cubic B-spline basis functions $\left(p \geq 3\right.$ ) providing $C^{2}$-continuity are adopted for spanning the approximation space.

By substituting (27) into (19), one can obtain the nonlinear equilibrium equations (after eliminating the arbitrary nodal virtual displacement $\delta \boldsymbol{d}_{I}$ ) as

$$
\boldsymbol{R}(\boldsymbol{d})=\sum_{I} \int_{0}^{L} \boldsymbol{B}_{I}^{T} \hat{\boldsymbol{\sigma}} d x-\boldsymbol{F}_{I}=0
$$

where $\boldsymbol{F}_{I}$ is the load vector

$$
\boldsymbol{F}_{I}=\int_{0}^{L} \phi_{I}\left[f_{x}, f_{y}\right]^{T} d x
$$

The nonlinear equation (29) is solved iteratively by the Newton-Raphson scheme in which the obtained solution is updated by an incremental displacement $\Delta \boldsymbol{d}$ given through the following system of a linear algebraic equation:

$$
\boldsymbol{K}_{T} \Delta \boldsymbol{d}=-\boldsymbol{R}
$$

where the tangent stiffness matrix is defined as

$$
\begin{aligned}
\boldsymbol{K}_{T} & =\frac{\partial \boldsymbol{R}}{\partial \boldsymbol{d}}=\sum_{I} \int_{0}^{L}\left(\boldsymbol{B}_{I}^{T} \frac{\partial \hat{\boldsymbol{\sigma}}}{\partial \boldsymbol{d}}+\frac{\partial \boldsymbol{B}_{I}^{T}}{\partial \boldsymbol{d}} \hat{\boldsymbol{\sigma}}\right) d x \\
& =\sum_{I} \sum_{J} \int_{0}^{L}\left(\boldsymbol{B}_{I}^{T} \boldsymbol{D} \boldsymbol{B}_{J}+N \phi_{I}^{\prime} \phi_{J}^{\prime} \boldsymbol{I}+R\left(\phi_{I}^{\prime} \phi_{J}^{\prime \prime}+\phi_{I}^{\prime \prime} \phi_{J}^{\prime}\right) \boldsymbol{I}\right) d x
\end{aligned}
$$

where $\boldsymbol{I}$ denotes an identity matrix. The iteration is repeated until the difference between two consecutive iterations reduces below a desired error tolerance, e.g., $0.1 \%$. For a detailed description of the solution procedure, one can refer to [Tran et al. 2015; Tran and Kim 2018].

\section{Numerical examples}

4.1. Model comparison. Let us consider a microbeam with thickness $h$, length $L$, and width $b=2 h$ subjected to a concentrated load $Q=100 \mu \mathrm{N}$ placed at the mid-span in the case of simply supported (SS) or clamped (CC) constraints at both ends or at the free-end of a cantilever beam (CF). At first, the assumption of small deformations is adopted for studying the linear behavior of the beams. It is assumed that the beam is made of epoxy with material properties as Young's modulus and Poisson ratio $E=1.44 \mathrm{GPa}$ and $v=0.38$, respectively, and with the length scale parameter assigned to be equal to $l=17.6 \mu \mathrm{m}$ [Lam et al. 2003]. Note that in the case of MSGT, the number of length scale parameters is reduced to one by setting $l_{0}=l_{1}=l_{2}=l=17.6 \mu \mathrm{m}$. The beam dimension is scaled up, by which the ratio 


\begin{tabular}{|r|rrcrccccc|}
\hline & \multicolumn{3}{|c}{ MSGT } & \multicolumn{2}{c}{ MCST } & \multicolumn{2}{c|}{ SSGT } & C \\
$h / l$ & FEM & $l$ & exact & $l$ & $l=33.08$ & $l$ & $l=19.91$ & exact \\
\hline SS 1 & 1.2718 & 1.2722 & 1.2722 & 3.8957 & 1.2733 & 1.601 & 1.270 & 20.8336 \\
5 & 12.8917 & 12.8998 & 12.8997 & 17.7471 & 12.9041 & 14.0603 & 12.8987 & 20.8336 \\
100 & 20.8013 & 20.8016 & 20.8013 & 20.8245 & 20.8016 & 20.8085 & 20.8016 & 20.8336 \\
\hline CC 1 & 0.3173 & 0.3173 & 0.3173 & 0.9739 & 0.3183 & 0.399 & 0.317 & 5.2083 \\
4 & 2.6526 & 2.6526 & 2.6526 & 4.0955 & 2.6571 & 2.9706 & 2.6515 & 5.2083 \\
8 & 4.1973 & 4.1973 & 4.1973 & 4.8771 & 4.2002 & 4.3829 & 4.1966 & 5.2083 \\
100 & 5.1999 & 5.2004 & 5.2003 & 5.2061 & 5.2004 & 5.2021 & 5.2004 & 5.2083 \\
\hline CF 1 & & 20.3678 & 20.3659 & 62.3306 & 20.372 & 25.6345 & 20.3667 & 333.333 \\
4 & & 170.0372 & 170.0373 & 262.1083 & 170.0555 & 190.4536 & 170.0324 & 333.333 \\
8 & & 268.7979 & 268.798 & 312.1289 & 268.8093 & 280.6895 & 268.7949 & 333.333 \\
100 & & 332.8219 & 332.8219 & 333.1885 & 332.822 & 332.9337 & 332.8219 & 333.333 \\
\hline
\end{tabular}

Table 1. Normalized central deflection $10^{3} \widehat{w}$ of the microbeams in the linear regime of deformation $(L / h=30, Q=100 \mu \mathrm{N}, E=$ $1.44 \mathrm{GPa}, v=0.38, l=17.6 \mu \mathrm{m}$, and $b=2 h$ ). See [Dadgar-Rad and Beheshti 2017] for the FEM column and [Timoshenko and Goodier 1970] for the C column.

of the thickness to the length scale parameter $h / l$ changes in the range of $[1,100]$, while the slenderness ratio is kept unchanged at the value $L / h=30$. The maximum normalized deflection $\widehat{w}=w E I /\left(Q L^{3}\right)$ for the three different types of beams based on MSGT, MCST, SSGT, and classical elasticity (C) has been reported in Table 1. For the sake of comparison, the results of Dadgar-Rad's work [Dadgar-Rad and Beheshti 2017] based on MSGT are inserted in the table. In addition, the maximum deflection values based on the classical Euler-Bernoulli beam theory [Timoshenko and Goodier 1970] given as $\widehat{w}=\frac{1}{48}, \frac{1}{192}, \frac{1}{3}$ for the simply supported, clamped, and cantilever beams, respectively, are also supplied. As can be seen, the deflections of classical elasticity are constant and independent of ratios $L / h$ and $h / l$. On the other hand, all nonclassical theories propose lower deflection values which are strongly dependent on ratio $h / l$. It is observed that the discrepancy as compared to the classical elasticity becomes very small as thickness $h$ is far greater than the value of the material length scale parameter $l$, e.g., $l / h=100$. Importantly, the present numerical simulation using IGA and based on only two unknowns ( $u$ and $w)$ produces results in accordance with the FEM results by Dadgar-Rad [Dadgar-Rad and Beheshti 2017] utilizing a nonconforming element with five degrees of freedom per node and also analytical solutions (given in the Appendix) for all of the case studies. For instance, as seen in Figure 1, the numerical results depicted with markers perfectly match the analytical curves plotted with lines. Moreover, 


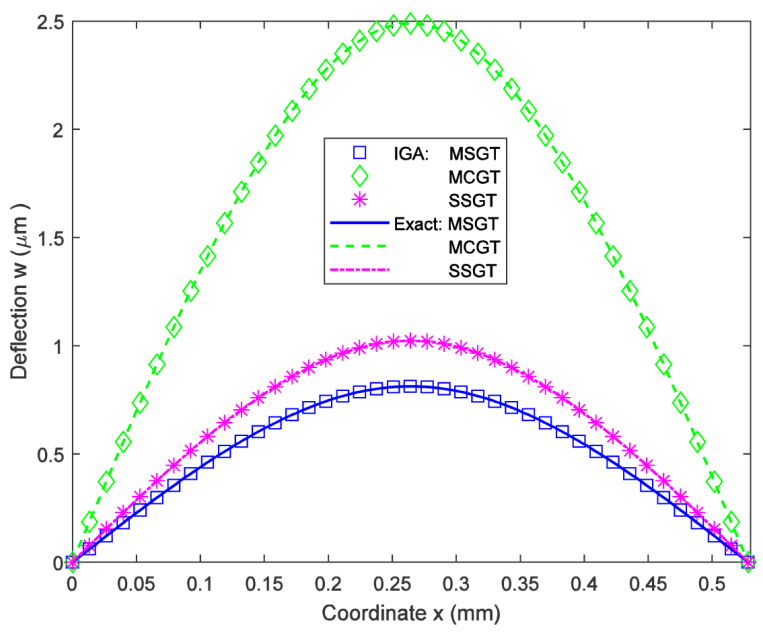

Figure 1. Comparison of various size-dependent theories for a simply supported beam $(L / h=30$ and $h=17.6 \mu \mathrm{m})$ with a concentrated load $Q=100 \mu \mathrm{N}$ at the mid-span. Note that the closed-form results are plotted with lines, while markers depict the numerical results based on IGA.

it is also clearly indicated that different strain gradient elasticity theories produce different results. For example, MCST in the green line, eliminating the sixth-order term in the governing equation (23), overestimates the transverse displacement as compared to the others. To find the relation between these nonclassical models, let us revisit the analytical deflection function in (35) with the values of integration constants given in (37), (39), and (41) according to the different types of boundary constraints. By keeping the integration constant $c_{3}$ fixed, a relation of the material length scale parameter for a particular model is given as

$$
\left(l^{\mathrm{MCST}}, l^{\mathrm{SSGT}}\right)=\left(\sqrt{\frac{53}{15}}, \sqrt{\frac{53}{30(1+v)}}\right) l^{\mathrm{MSGT}} .
$$

According to relation (33), the material length scale parameters of MCST and SSGT are given by factors 33.08 and 19.91, respectively, in accordance with the parameter 17.6 of MSGT. With these factors, the results of the models become practically identical as seen in Table 1 [Niiranen et al. 2019].

As the next step, we again investigate the singly simply supported microbeam with the above data except for a couple of changes: the slenderness ratio is $L / h=$ 20 and the concentrated load is $Q=12 \mathrm{mN}$, in order to make sure that the beam exhibits a relatively large deflection involving geometric nonlinearity. Table 2 lists a tabular comparison between the nonlinear finite element analysis by Dadgar-Rad [Dadgar-Rad and Beheshti 2017] based on the MSGT and classical elasticity and 


\begin{tabular}{|r|rrrrrrrrr|}
\hline & \multicolumn{2}{|c}{ MSGT } & \multicolumn{2}{c}{ MCST } & \multicolumn{2}{c|}{ SSGT } & \multicolumn{2}{c|}{ classical } \\
$h / l$ & \multicolumn{1}{|c}{ FEM } & $l$ & $l$ & $l=33.08$ & $l$ & $l=19.91$ & \multicolumn{1}{c|}{ FEM } & \multicolumn{1}{c|}{ IGA } \\
\hline 1 & 0.9895 & 0.9897 & 1.4253 & 0.9909 & 1.1065 & 0.9894 & 1.7312 & 1.6765 \\
2 & 2.8646 & 2.8649 & 3.6020 & 2.8675 & 3.0727 & 2.8643 & 4.0287 & 3.9591 \\
4 & 7.1612 & 7.1622 & 8.2773 & 7.1663 & 7.4860 & 7.1611 & 8.8529 & 8.7573 \\
100 & 20.8010 & 20.8058 & 20.8287 & 20.8058 & 20.8124 & 20.8054 & 21.0016 & 20.8331 \\
\hline
\end{tabular}

Table 2. Normalized central deflection $10^{3} \widehat{w}$ of a simply supported microbeams considering geometrically nonlinear effect under a concentrated load at mid-span $Q=12 \mathrm{mN}(L / h=20$, $E=1.44 \mathrm{GPa}, v=0.38, l=17.6 \mu \mathrm{m}, b=2 h)$. See [DadgarRad and Beheshti 2017] for the FEM columns.

the present IGA. In addition, we also provide some results using MCST, SSGT, and classical elasticity as well, for comparison. First, let us note that the normalized deflection reduces with an increase in thickness, in contrast to the constant 20.8336 of the classical model. As seen, the smallest beam $(h=l)$ exhibits the largest deformation with relative deflection $w / h=2.165$. Thus, the geometrically nonlinear effect becomes significant and makes the beam stiffer. Meanwhile, the largest beam $(h=100 l)$ reveals a very small relative deflection $w / h=0.003$, indicating no geometrically nonlinear effects. Therefore, the transverse displacement coincides with that of linear analysis - opposite to the finite element method used by Dadgar-Rad [Dadgar-Rad and Beheshti 2017] which overestimates the linear solution. This indicates that the present nonlinear finite element formulation works very well for both linear and nonlinear bending analysis. Furthermore, the same conclusions regarding linear analysis are drawn here. (1) The obtained results are in good agreement with the result using FEM in [Dadgar-Rad and Beheshti 2017], and (2) the nonclassical theories exhibit higher bending rigidity due to the appearance of gradient terms related to the material length scale parameter $l$. However, the effect is no longer dominant in large-scale structures (e.g., $h / l=100$ ). (3) By choosing the material length scale parameter according to (33), the results are practically identical for all the generalized theories. Therefore, in future studies, we prefer to use MSGT as a representative model in the numerical simulations unless otherwise specified.

\subsection{Nonlinear behavior of generalized beams under a uniform distributed load.}

Let us continue to study the nonlinear behavior of the microbeams with thickness $h=17.6 \mathrm{~mm}$ and slenderness ratio $L / h=20$ subjected to a uniform distributed load with magnitude $q=30 \mathrm{~N} / \mathrm{m}$ for the simply supported (SS) beam or $q=60 \mathrm{~N} / \mathrm{m}$ if the beam is clamped (CC). These loading values are chosen to make sure that the maximum rotation of the cross-section does not exceed $15^{\circ}$ (Figures 3 and 4 ), 

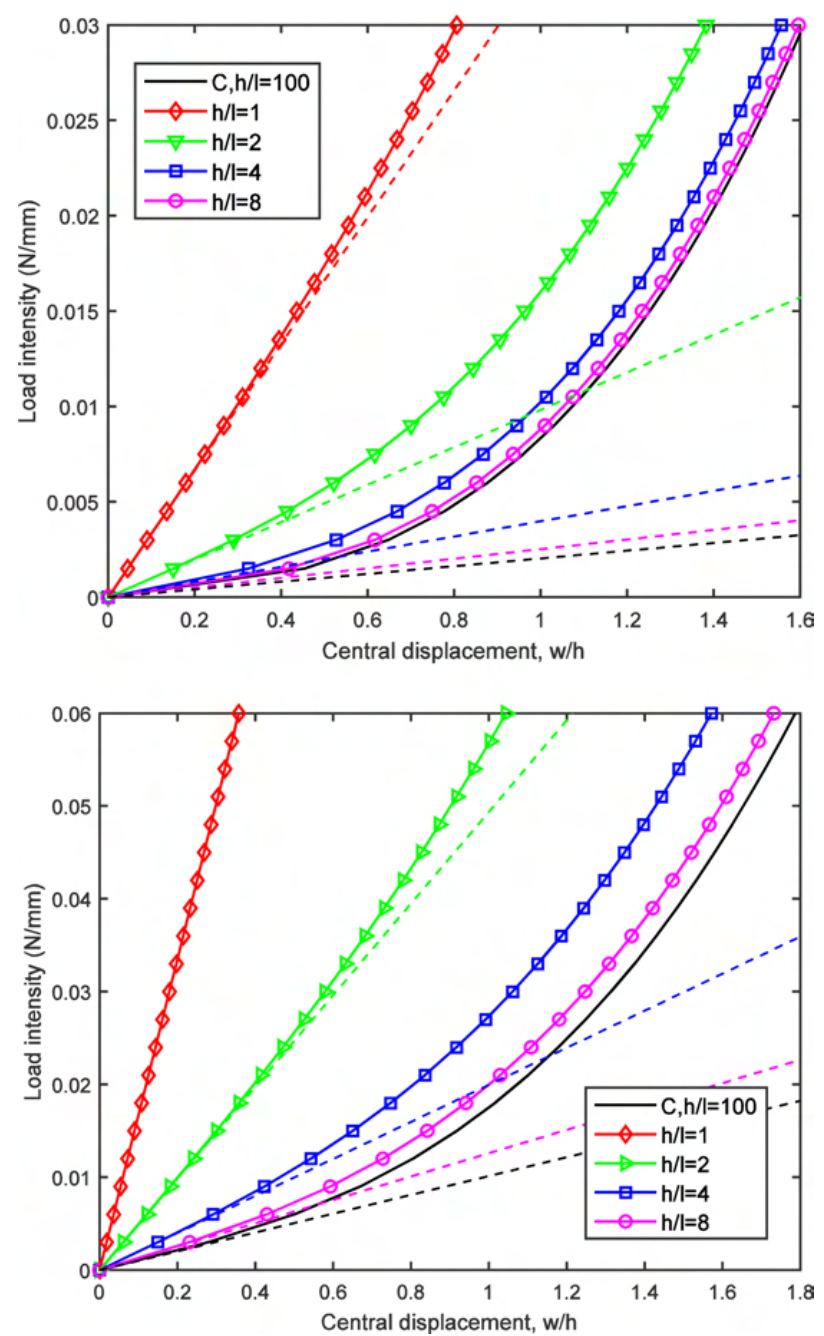

Figure 2. Load displacement curve of a microbeam under different boundary conditions: (bottom) SS and (top) CC (with linear and nonlinear responses plotted with dashed and solid lines, respectively).

which satisfies the moderate rotation limitation of the von Kármán assumption. Figure 2 plots the load displacement curves at the mid-span of the beams for different values of the length scale parameter $l$ scaled down to $[1,2,4,8,100]$ times thickness $h$. It is observed that the linear load displacement responses (dashed lines) are always tangent - at the origin — to the nonlinear load displacement curves (solids lines). Also, the response curves are strongly dependent on ratio $h / l$. As can be seen, the stiffest beam $(h / l=1)$ does not exhibit much nonlinearity, typically 

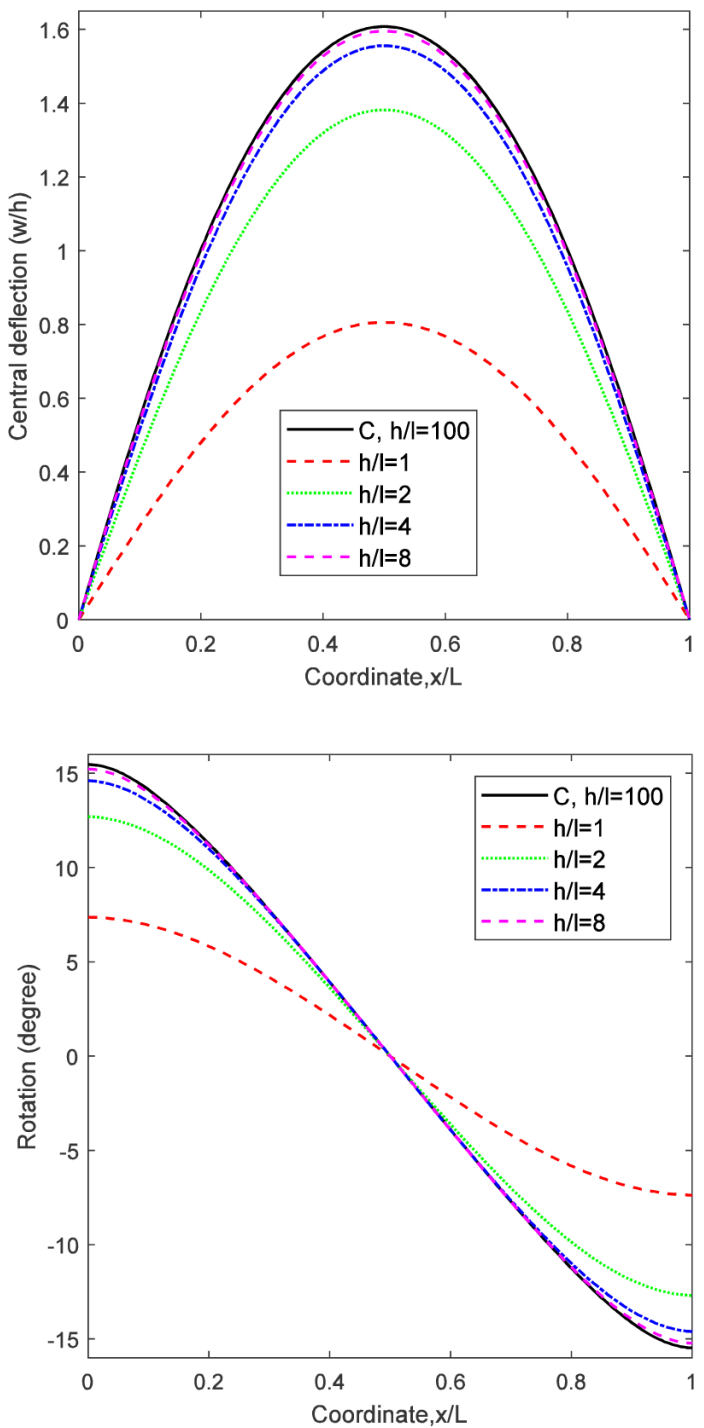

Figure 3. Behavior of a simply supported beam under a uniform load $q=30 \mathrm{~N} / \mathrm{m}$ with $L / h=20$ and $h=17.6 \mu \mathrm{m}$ : (top) the deflection profile and (bottom) the corresponding rotation of the cross-section.

meaning that the linear and nonlinear results coincide (especially for the clamped beam). However, increase in ratio $h / l$ essentially increases the beam deflection. As the deflection-to-thickness ratio becomes high (i.e., $w / h>1$ ), geometric nonlinearity plays a more essential role through the large difference between the linear and nonlinear solutions. To close this subsection, the distributions of transverse 

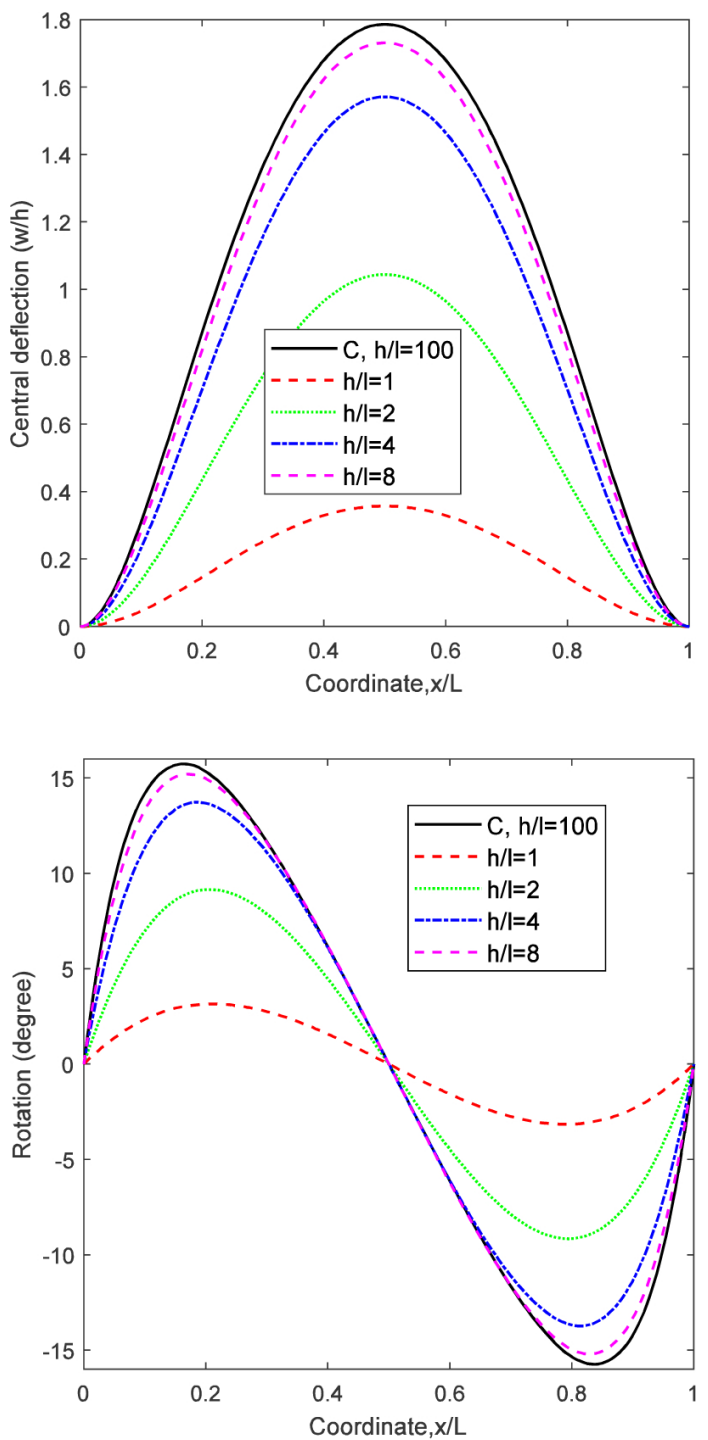

Figure 4. Behavior of a clamped-clamped beam under a uniform load $q=60 \mathrm{~N} / \mathrm{m}$ with $L / h=20$ and $h=17.6 \mu \mathrm{m}$ : (top) the deflection profile and (bottom) the corresponding rotation of the cross-section.

displacement and cross-section rotations through the beam axis coordinate are revealed in Figures 3 and 4. It is noted that the absolute value of rotation based on the strain gradient theory is smaller than that predicted by the classical formulation due to the size effect taken into account. 


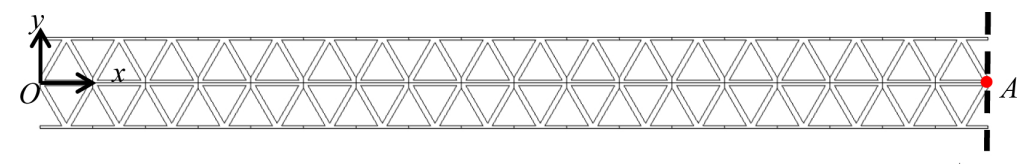

symmetry

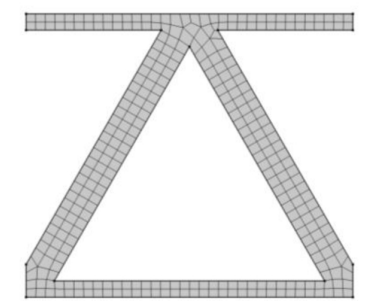

Figure 5. Top: one half of a lattice frame with $L=90 \mathrm{~mm}$ and $h=8.66 \mathrm{~mm}$, produced by replicating a unit cell 18 and 2 times along the $x$-and $y$-axes, respectively. Bottom: 2D finite element mesh of a unit cell in the FEM software COMSOL Multiphysics.

4.3. Application to triangular lattice structures. In order to demonstrate the applicability of strain gradient theories for structures, we further study the linear and geometrically nonlinear behavior of an elastic triangular lattice frame (see Figure 5) with length $L=180 \mathrm{~mm}$ and height $h=8.66 \mathrm{~mm}$. The frame is constrained at two ends by clamped and simply supported conditions and subjected to a uniformly distributed load applied in increments of $\Delta q=4 \mathrm{~N} / \mathrm{m}$ until reaching the final magnitude of $200 \mathrm{~N} / \mathrm{m}$. Due to symmetry, only a half of the frame is modeled as given in Figure 5, top. As seen, the lattice strip can be produced simply by replicating a unit cell or the so-called representative volume element (RVE with the dimensions from [Khakalo et al. 2018, Table 3]). The material properties of the structure are simply Young's modulus $E=2 \mathrm{GPa}$ and Poisson's ratio $v=0.25$.

A reference model of the structure is built by using linear quadrilateral finite elements of classical elasticity in COMSOL Multiphysics with a mesh for each unit cell shown in Figure 5, bottom. The structure can be modeled by a one-dimensional generalized beam model as a homogenized isotropic beam with equivalent mechanical properties as $E_{\text {eff }}=246.7 \mathrm{MPa}$ and $\nu_{\text {eff }}=0.335$ and intrinsic material length scale parameter $l=1.57 \mathrm{~mm}$ [Khakalo et al. 2018].

The maximum deflection of the beam is recorded at the mid-span and plotted for each load increment in Figure 6. The red solid lines denote the nonlinear solutions, while red dashed lines correspond to the linear ones, both corresponding to the 2D reference model. As seen, the present results from the 1D strain gradient beam model with red circles and diamond markers, corresponding to the linear and nonlinear regimes, respectively, are nearly lying on the curves. This means that the strain gradient beam model captures the bending behavior of the lattice 

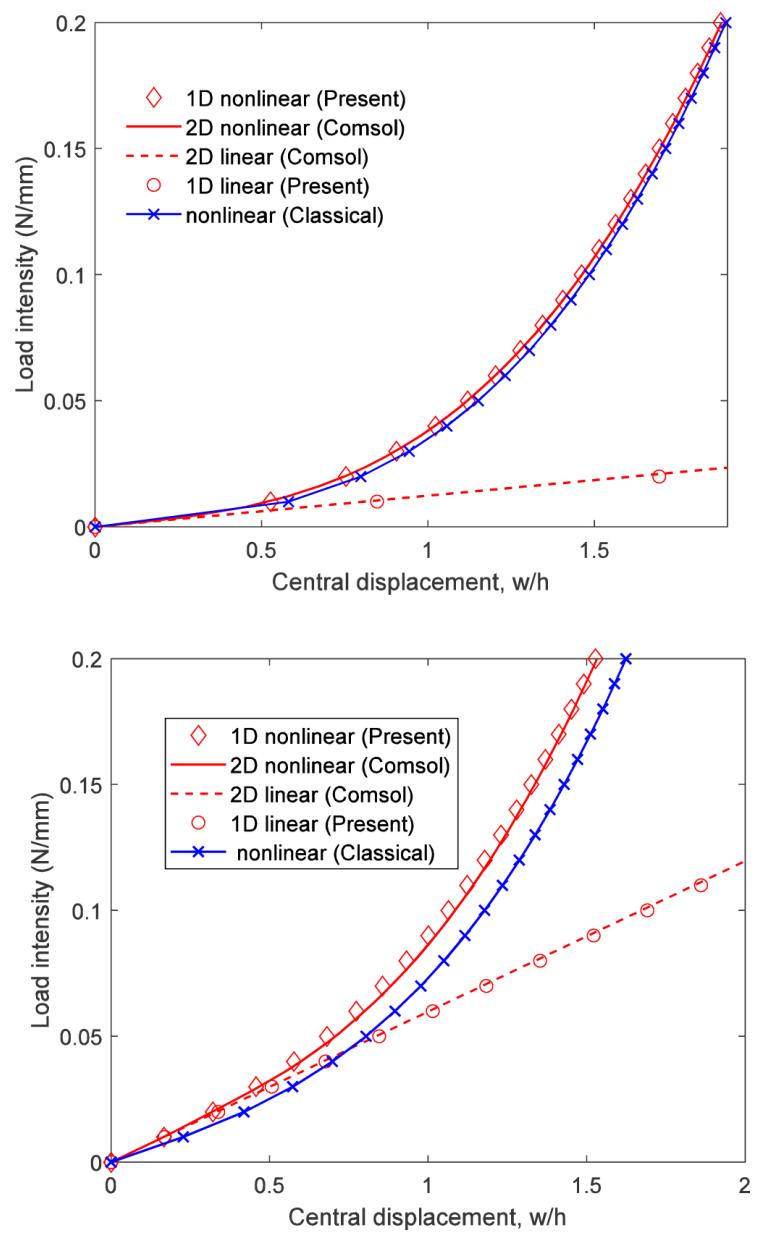

Figure 6. Load displacement curves of a lattice beam under uniform load $q=200 \mathrm{~N} / \mathrm{m}$ with $h=8.66 \mathrm{~mm}, L=180 \mathrm{~mm}$, and $l=1.57 \mathrm{~mm}$ with (top) simply supported and (bottom) clamped boundary conditions.

frame in both linear and nonlinear regimes. Again, the same observation as for Figure 2 is that the predicted deflection of the lattice frame (or the strain gradient beam model) is always smaller than that of the classical beam theory plotted with a blue curve. Furthermore, a comparison of the deflection distribution between the 2D reference and 1D beam simulations is plotted in Figure 7. As observed, the present generalized beam model is in good accordance with the global deflection of the frame represented through the mid-line OA but, naturally, does not describe in detail any local behavior such as bending or buckling of single struts near the clamped end of the frame as shown in Figure 8, bottom. 


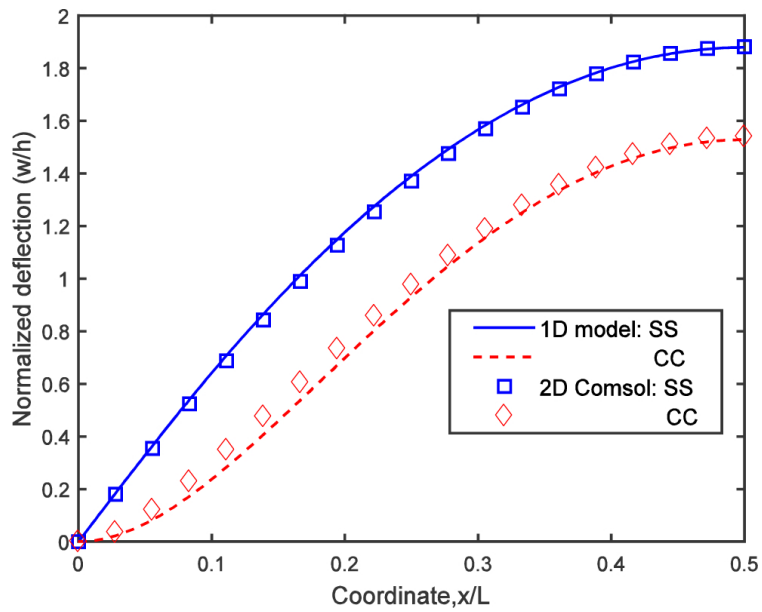

Figure 7. A comparison for the deflection distribution along the mid-line between the $1 \mathrm{D}$ beam model and $2 \mathrm{D}$ reference simulations with clamped (CC) and simply supported (SS) boundary conditions.

\begin{tabular}{|l|rrrr|}
\hline & \multicolumn{2}{|c}{ 2D reference model } & \multicolumn{2}{c|}{ 1D beam model } \\
$N$ & DoFs & time (s) & DoFs & time (s) \\
\hline 2 & 165366 & 258 & & \\
4 & 651562 & 880 & 24 & 0.5 \\
8 & 2586450 & 4416 & & \\
\hline
\end{tabular}

Table 3. Number of DoFs and time consumption for the present 1D beam model and the 2D reference model.

To end this subsection, we discuss the computational efficiency of the present beam model by studying clamped lattice structure strips subjected to a concentrated load $P=30 \mathrm{~N}$ at the mid-span. A series of beam-like lattice structures is formed by scaling up the frame studied above having two $(N=2)$ unit cells in the thickness direction. The subsequent structures of the series have four and eight $(N=4$ and $N=8$ ) unit cells in the thickness direction, whereas the slenderness of the beam-like structures is kept constant: $L / h=20.7$. Table 3 shows the number of degrees of freedom (DoFs) and time consumption in the 2D reference and 1D beam simulations. As seen, in 2D simulations doubling factor $N$ implies an increase of $N^{2}$ DoFs. Meanwhile, the present strain gradient beam model requires 8 elements associated with $24 \mathrm{DoFs}$ (as using quartic B-spline basic functions with $q=4$ ) but still achieves a good agreement with the 2D solutions as shown in Figure 9. (Both programs are compiled on a desktop PC with an Intel Core i7-7600U $2.80 \mathrm{GHz}$ CPU and 16 GB of RAM.) 

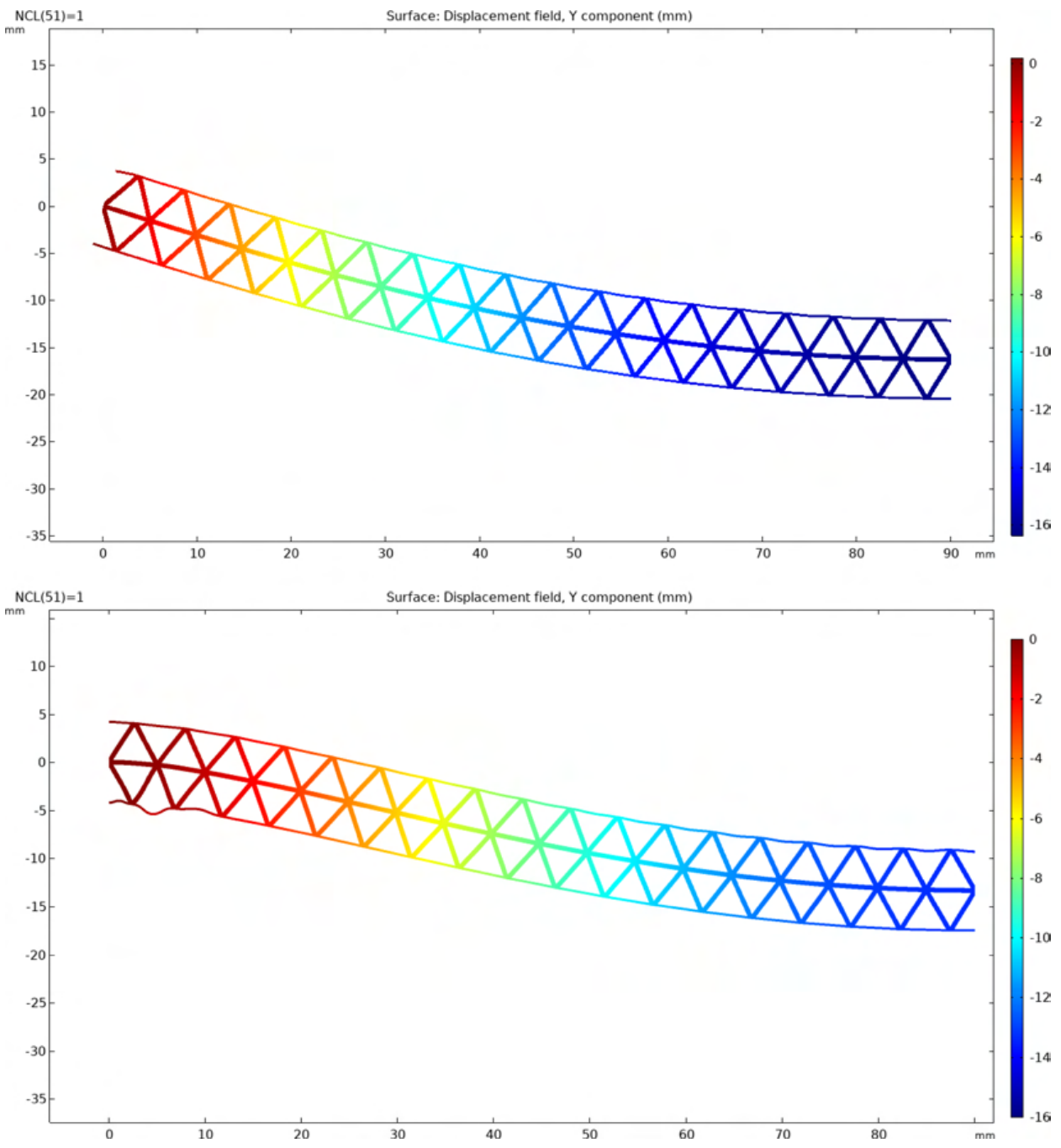

Figure 8. The transverse displacement of a lattice frame modeled by $2 \mathrm{D}$ finite elements (with COMSOL Multiphysics) under simply supported (top) and clamped (bottom) boundary conditions.

\section{Conclusions}

This paper studies the nonlinear bending of the Euler-Bernoulli beam model within Mindlin's strain gradient elasticity theory of form II retrieved with simplified oneparameter beam models of generalized elasticity. In principle, different beam models give different results. In practice, however, by choosing the value of the length scale parameter properly, almost identical results can be obtained - for both linear and nonlinear regimes of deformation. Furthermore, the geometrical nonlinearity 


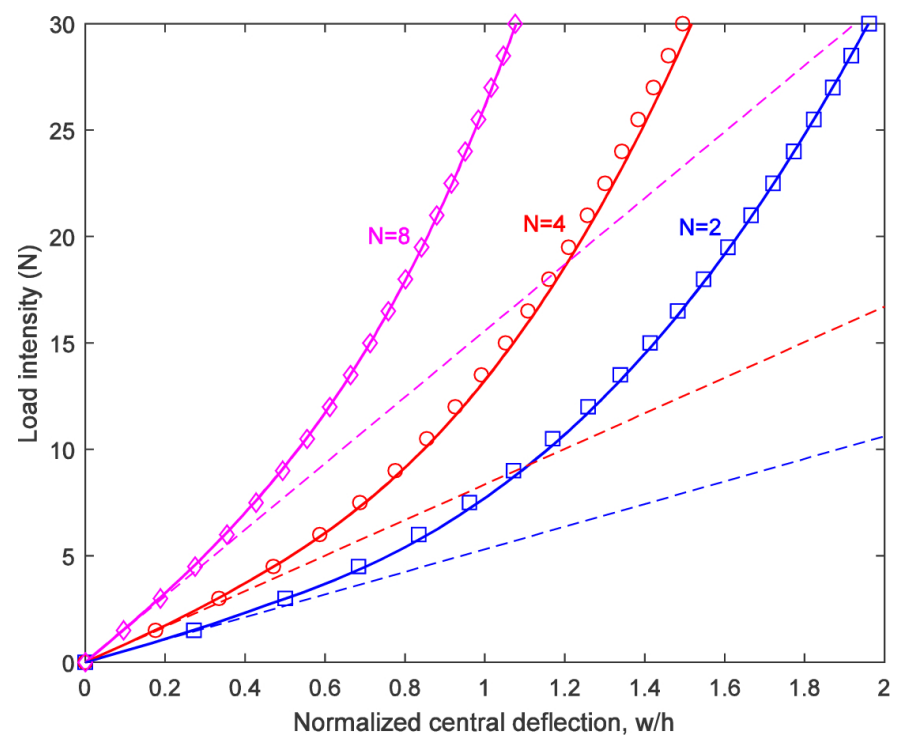

Figure 9. Load displacement curves for structures with $N$ unit cells in the thickness direction: linear solutions (dashed lines), nonlinear solutions with 2D reference model (solid lines), and 1D beam model (markers).

and size effects reduce the deflection of the beams as compared to the classical theory of elasticity, especially as the material length scale parameter becomes comparable to the beam thickness. It is noted that according to the von Kármán strain assumptions nonlinear deformations are limited to small or moderate rotations. More general large deformations are left for further works.

For the computational part, besides analytical results for some benchmark problems, a conforming and isogeometric B-spline Galerkin discretization is adopted for numerical solutions. With basis functions of order $p \geq 3$, the method naturally satisfies the stringent $C^{2}$-continuity required by the strain gradient beam model.

Finally, we demonstrate the advantage of the present beam model by studying 2D lattice frame structures. With a $1 \mathrm{D}$ beam model, we significantly reduce the number of DoFs but still maintain a good level of accuracy as compared with 2D reference simulations. However, the beam model as a homogenized model describing the mid-line of the beam does not describe such behavior as local bending or buckling of single lattice struts in the vicinity of concentrated loads or constrained boundaries. A homogenization method considering initial imperfections for geometrically nonlinear analysis [Reinaldo Goncalves et al. 2016] could be considered to treat this issue in future studies. 


\section{Appendix: Analytical solution for linear statics of strain-gradient-elastic thin beams}

First, let us consider a singly simply supported beam with a concentrated load $Q$ at the mid-span. The governing equation related to the transverse displacement can be derived from (23) by setting $f_{y}=0$, giving

$$
\left(E I+\alpha_{2} A\right) w^{(4)}-\alpha_{1} I w^{(6)}=0 .
$$

The analytical solution of (34) is given by

$$
w(x)=c_{0}+c_{1} x+c_{2} x^{2}+c_{3} x^{3}+c_{4} e^{\beta x}+c_{5} e^{-\beta x}
$$

in which $\beta=\sqrt{\left(E I+\alpha_{2} A\right) / \alpha_{1} I}$ and the six integration constants $c_{I}(I=0,1, \ldots, 5)$ are determined from the essential and natural boundary conditions. In this beam problem, due to symmetry, a half of the beam is considered with boundary conditions

$$
\begin{aligned}
w(0) & =0, & \bar{V}(L / 2) & =Q / 2, \\
\bar{M}(0) & =0, & w^{\prime}(L / 2) & =0, \\
w^{\prime \prime \prime}(0) & =0, & w^{\prime \prime \prime}(L / 2) & =0 .
\end{aligned}
$$

With these constrains, the integration constants are defined as

$$
\begin{array}{ll}
c_{0}=\frac{6 c_{3}}{\beta^{3} \sinh (\beta L / 2)}(1-\cosh (\beta L / 2)), & c_{3}=\frac{-Q}{12\left(E I+\alpha_{2} A\right)}, \\
c_{1}=c_{3}\left(\frac{6}{\beta^{2}}-\frac{3 L^{2}}{4}\right), & c_{4}=\frac{-3 c_{3}}{\beta^{3} \sinh (\beta L / 2)}\left(1-e^{-\beta L / 2}\right), \\
c_{2}=0, & c_{5}=\frac{-3 c_{3}}{\beta^{3} \sinh (\beta L / 2)}\left(1-e^{\beta L / 2}\right) .
\end{array}
$$

Second, analogously, a singly clamped beam with a concentrated load at the mid-span satisfies the boundary conditions

$$
\begin{aligned}
w(0) & =0, & \bar{V}(L / 2) & =Q / 2, \\
w^{\prime}(0) & =0, & w^{\prime}(L / 2) & =0, \\
w^{\prime \prime \prime}(0) & =0, & w^{\prime \prime \prime}(L / 2) & =0 .
\end{aligned}
$$


The integration constants are accordingly defined as

$$
\begin{aligned}
c_{0} & =\frac{6 c_{3}}{\beta^{3} \sinh (\beta L / 2)}(1-\cosh (\beta L / 2)), & c_{3} & =\frac{-Q}{12\left(E I+\alpha_{2} A\right)}, \\
c_{1} & =\frac{6 c_{3}}{\beta^{2}}, & c_{4} & =\frac{-3 c_{3}}{\beta^{3} \sinh (\beta L / 2)}\left(1-e^{-\beta L / 2}\right), \\
c_{2} & =-\frac{3 L c_{3}}{4}, & c_{5} & =\frac{-3 c_{3}}{\beta^{3} \sinh (\beta L / 2)}\left(1-e^{\beta L / 2}\right) .
\end{aligned}
$$

Third, a cantilever beam subjected to loading $Q$ at the free end gets the integration constants of (41) which satisfy the boundary conditions

$$
\begin{aligned}
w(0) & =w^{\prime}(0)=0, \\
w^{\prime \prime \prime}(0) & =w^{\prime \prime \prime}(L)=0, \\
\bar{V}(L) & =Q, \quad \bar{M}(L)=0
\end{aligned}
$$

and integration constants

$$
\begin{aligned}
c_{0} & =\frac{6 c_{3}}{\beta^{3} \sinh (\beta L)}(1-\cosh (\beta L)), & c_{3} & =\frac{-Q}{6\left(E I+\alpha_{2} A\right)}, \\
c_{1} & =6 c_{3} / \beta^{2}, & c_{4} & =\frac{-3 c_{3}}{\beta^{3} \sinh (\beta L)}\left(1-e^{-\beta L}\right), \\
c_{2} & =-3 c_{3} L, & c_{5} & =\frac{-3 c_{3}}{\beta^{3} \sinh (\beta L)}\left(1-e^{\beta L}\right),
\end{aligned}
$$

\section{References}

[Abdel-Rahman et al. 2002] E. M. Abdel-Rahman, M. I. Younis, and A. H. Nayfeh, "Characterization of the mechanical behavior of an electrically actuated microbeam", J. Micromech. Microeng. 12:6 (2002), 759-766.

[Abdoul-Anziz and Seppecher 2018] H. Abdoul-Anziz and P. Seppecher, "Strain gradient and generalized continua obtained by homogenizing frame lattices", Math. Mech. Complex Syst. 6:3 (2018), 213-250.

[Aifantis 1992] E. C. Aifantis, "On the role of gradients in the localization of deformation and fracture”, Internat. J. Engrg. Sci. 30:10 (1992), 1279-1299.

[Altan and Aifantis 1997] B. S. Altan and E. C. Aifantis, "On some aspects in the special theory of gradient elasticity”, J. Mech. Behav. Mat. 8:3 (1997), 231-282.

[Asghari et al. 2010] M. Asghari, M. H. Kahrobaiyan, and M. T. Ahmadian, "A nonlinear Timoshenko beam formulation based on the modified couple stress theory", Internat. J. Engrg. Sci. 48:12 (2010), 1749-1761.

[Asghari et al. 2012] M. Asghari, M. H. Kahrobaiyan, M. Nikfar, and M. T. Ahmadian, "A sizedependent nonlinear Timoshenko microbeam model based on the strain gradient theory", Acta Mech. 223:6 (2012), 1233-1249.

[Askes and Aifantis 2009] H. Askes and E. C. Aifantis, "Gradient elasticity and flexural wave dispersion in carbon nanotubes", Phys. Rev. B 80:19 (2009), art. id. 195412. 
[Balobanov and Niiranen 2018] V. Balobanov and J. Niiranen, "Locking-free variational formulations and isogeometric analysis for the Timoshenko beam models of strain gradient and classical elasticity", Comput. Methods Appl. Mech. Engrg. 339 (2018), 137-159.

[Bauer et al. 2016] A. M. Bauer, M. Breitenberger, B. Philipp, R. Wüchner, and K.-U. Bletzinger, "Nonlinear isogeometric spatial Bernoulli beam", Comput. Methods Appl. Mech. Engrg. 303 (2016), 101-127.

[Boutin et al. 2017] C. Boutin, F. dell'Isola, I. Giorgio, and L. Placidi, "Linear pantographic sheets: asymptotic micro-macro models identification”, Math. Mech. Complex Syst. 5:2 (2017), 127-162.

[Chang et al. 2007] W.-J. Chang, H.-L. Lee, and T. Y.-F. Chen, "Study of the sensitivity of the first four flexural modes of an AFM cantilever with a sidewall probe", Ultramicroscopy 108:7 (2007), 619-624.

[Cosserat and Cosserat 1909] E. Cosserat and F. Cosserat, Théorie des corps déformables, Hermann, Paris, 1909.

[Dadgar-Rad and Beheshti 2017] F. Dadgar-Rad and A. Beheshti, "A nonlinear strain gradient finite element for microbeams and microframes”, Acta Mech. 228:5 (2017), 1941-1964.

[dell'Isola et al. 2016] F. dell'Isola, A. Della Corte, I. Giorgio, and D. Scerrato, "Pantographic 2D sheets: discussion of some numerical investigations and potential applications", Int. J. Nonlin. Mech. 80 (2016), 200-208.

[dell'Isola et al. 2019a] F. dell'Isola, P. Seppecher, J. J. Alibert, and et al., "Pantographic metamaterials: an example of mathematically driven design and of its technological challenges", Contin. Mech. Thermodyn. 31:4 (2019), 851-884.

[dell'Isola et al. 2019b] F. dell'Isola, P. Seppecher, M. Spagnuolo, E. Barchiesi, F. Hild, T. Lekszycki, I. Giorgio, L. Placidi, U. Andreaus, M. Cuomo, S. R. Eugster, A. Pfaff, K. Hoschke, R. Langkemper, E. Turco, R. Sarikaya, A. Misra, M. De Angelo, F. D’Annibale, A. Bouterf, X. Pinelli, A. Misra, B. Desmorat, M. Pawlikowski, C. Dupuy, D. Scerrato, P. Peyre, M. Laudato, L. Manzari, P. Göransson, C. Hesch, S. Hesch, P. Franciosi, J. Dirrenberger, F. Maurin, Z. Vangelatos, C. Grigoropoulos, V. Melissinaki, M. Farsari, W. Muller, B. E. Abali, C. Liebold, G. Ganzosch, P. Harrison, R. Drobnicki, L. Igumnov, F. Alzahrani, and T. Hayat, "Advances in pantographic structures: design, manufacturing, models, experiments and image analyses", Contin. Mech. Thermodyn. 31:4 (2019), $1231-1282$.

[Eringen 1999] A. C. Eringen, "Theory of micropolar elasticity”, Chapter 5, pp. 101-248 in Microcontinuum field theories, vol. I: Foundations and solids, Springer, 1999.

[Fleck et al. 1994] N. A. Fleck, G. M. Muller, M. F. Ashby, and J. W. Hutchinson, "Strain gradient plasticity: theory and experiment", Acta. Metall. Mater. 42:2 (1994), 475-487.

[Greco 2020] L. Greco, "An iso-parametric $\mathrm{G}^{1}$-conforming finite element for the nonlinear analysis of Kirchhoff rod, I: The 2D case”, Contin. Mech. Thermodyn. 32:5 (2020), 1473-1496.

[Greco and Cuomo 2014] L. Greco and M. Cuomo, "An implicit $G^{1}$ multi patch B-spline interpolation for Kirchhoff-Love space rod”, Comput. Methods Appl. Mech. Engrg. 269 (2014), 173-197.

[Greco et al. 2017] L. Greco, M. Cuomo, L. Contrafatto, and S. Gazzo, "An efficient blended mixed B-spline formulation for removing membrane locking in plane curved Kirchhoff rods", Comput. Methods Appl. Mech. Engrg. 324 (2017), 476-511.

[Green and Rivlin 1964] A. E. Green and R. S. Rivlin, "Multipolar continuum mechanics", Arch. Rational Mech. Anal. 17:2 (1964), 113-147.

[Hassanpour et al. 2010] P. A. Hassanpour, E. Esmailzadeh, W. L. Cleghorn, and J. K. Mills, "Nonlinear vibration of micromachined asymmetric resonators", J. Sound Vib. 329:13 (2010), 25472564. 
[Hu et al. 2004] Y. C. Hu, C. M. Chang, and S. C. Huang, "Some design considerations on the electrostatically actuated microstructures”, Sensor. Actuator. A Phys. 112:1 (2004), 155-161.

[Hughes et al. 2005] T. J. R. Hughes, J. A. Cottrell, and Y. Bazilevs, "Isogeometric analysis: CAD, finite elements, NURBS, exact geometry and mesh refinement", Comput. Methods Appl. Mech. Engrg. 194:39-41 (2005), 4135-4195.

[Kahrobaiyan et al. 2011] M. H. Kahrobaiyan, M. Asghari, M. Rahaeifard, and M. T. Ahmadian, "A nonlinear strain gradient beam formulation", Internat. J. Engrg. Sci. 49:11 (2011), 1256-1267.

[Khakalo and Niiranen 2018] S. Khakalo and J. Niiranen, "Form II of Mindlin's second strain gradient theory of elasticity with a simplification: for materials and structures from nano- to macroscales", Eur. J. Mech. A Solids 71 (2018), 292-319.

[Khakalo and Niiranen 2019] S. Khakalo and J. Niiranen, "Lattice structures as thermoelastic strain gradient metamaterials: evidence from full-field simulations and applications to functionally stepwise-graded beams", Compos. Part B Eng. 177 (2019), art. id. 107224.

[Khakalo and Niiranen 2020] S. Khakalo and J. Niiranen, "Anisotropic strain gradient thermoelasticity for cellular structures: plate models, homogenization and isogeometric analysis", J. Mech. Phys. Solids 134 (2020), art. id. 103728.

[Khakalo et al. 2018] S. Khakalo, V. Balobanov, and J. Niiranen, "Modelling size-dependent bending, buckling and vibrations of 2D triangular lattices by strain gradient elasticity models: applications to sandwich beams and auxetics", Internat. J. Engrg. Sci. 127 (2018), 33-52.

[Kiendl et al. 2015] J. Kiendl, F. Auricchio, T. J. R. Hughes, and A. Reali, "Single-variable formulations and isogeometric discretizations for shear deformable beams", Comput. Methods Appl. Mech. Engrg. 284 (2015), 988-1004.

[Kong et al. 2009] S. Kong, S. Zhou, Z. Nie, and K. Wang, "Static and dynamic analysis of micro beams based on strain gradient elasticity theory", Internat. J. Engrg. Sci. 47:4 (2009), 487-498.

[Lam et al. 2003] D. C. C. Lam, F. Yang, A. C. M. Chong, J. Wang, and P. Tonga, "Experiments and theory in strain gradient elasticity", J. Mech. Phys. Solids 51:8 (2003), 1477-1508.

[Lazar and Po 2015] M. Lazar and G. Po, "The non-singular Green tensor of Mindlin's anisotropic gradient elasticity with separable weak non-locality", Phys. Lett. A 379:24-25 (2015), 1538-1543.

[Lazopoulos 2004] K. A. Lazopoulos, "On the gradient strain elasticity theory of plates", Eur. J. Mech. A Solids 23:5 (2004), 843-852.

[Lazopoulos 2012] A. K. Lazopoulos, "Dynamic response of thin strain gradient elastic beams", Int. J. Mech. Sci. 58:1 (2012), 27-33.

[Lazopoulos and Lazopoulos 2010] K. A. Lazopoulos and A. K. Lazopoulos, "Bending and buckling of thin strain gradient elastic beams", Eur. J. Mech. A Solids 29:5 (2010), 837-843.

[Lazopoulos and Lazopoulos 2011] K. A. Lazopoulos and A. K. Lazopoulos, "Nonlinear strain gradient elastic thin shallow shells", Eur. J. Mech. A Solids 30:3 (2011), 286-292.

[Lazopoulos et al. 2014] A. K. Lazopoulos, K. A. Lazopoulos, and G. Palassopoulos, "Nonlinear bending and buckling for strain gradient elastic beams", Appl. Math. Model. 38:1 (2014), 253-262.

[Lun et al. 2006] F.-y. Lun, P. Zhang, F.-b. Gao, and H.-g. Jia, "Design and fabrication of microopto-mechanical vibration sensor”, Microfab. Tech. 2006:1 (2006), 61-64.

[Lurie and Solyaev 2018] S. Lurie and Y. Solyaev, "Revisiting bending theories of elastic gradient beams", Internat. J. Engrg. Sci. 126 (2018), 1-21.

[Luu et al. 2015] A.-T. Luu, N.-I. Kim, and J. Lee, "Bending and buckling of general laminated curved beams using NURBS-based isogeometric analysis", Eur. J. Mech. A Solids 54 (2015), 218231. 
[Ma et al. 2008] H. M. Ma, X.-L. Gao, and J. N. Reddy, "A microstructure-dependent Timoshenko beam model based on a modified couple stress theory", J. Mech. Phys. Solids 56:12 (2008), 33793391.

[Mindlin 1964] R. D. Mindlin, "Micro-structure in linear elasticity", Arch. Rational Mech. Anal. 16 (1964), 51-78.

[Mindlin and Eshel 1968] R. D. Mindlin and N. N. Eshel, "On first strain-gradient theories in linear elasticity”, Int. J. Solids Struct. 4:1 (1968), 109-124.

[Moghimi Zand and Ahmadian 2009] M. Moghimi Zand and M. T. Ahmadian, "Vibrational analysis of electrostatically actuated microstructures considering nonlinear effects", Commun. Nonlinear Sci. Numer. Sim. 14:4 (2009), 1664-1678.

[Niiranen et al. 2016] J. Niiranen, S. Khakalo, V. Balobanov, and A. H. Niemi, "Variational formulation and isogeometric analysis for fourth-order boundary value problems of gradient-elastic bar and plane strain/stress problems", Comput. Methods Appl. Mech. Engrg. 308 (2016), 182-211.

[Niiranen et al. 2017] J. Niiranen, J. Kiendl, A. H. Niemi, and A. Reali, "Isogeometric analysis for sixth-order boundary value problems of gradient-elastic Kirchhoff plates", Comput. Methods Appl. Mech. Engrg. 316 (2017), 328-348.

[Niiranen et al. 2019] J. Niiranen, V. Balobanov, J. Kiendl, and S. B. Hosseini, "Variational formulations, model comparisons and numerical methods for Euler-Bernoulli micro- and nano-beam models", Math. Mech. Solids 24:1 (2019), 312-335.

[Park and Gao 2006] S. K. Park and X.-L. Gao, "Bernoulli-Euler beam model based on a modified couple stress theory", J. Micromech. Microeng. 16:11 (2006), 2355-2359.

[Piegl and Tiller 1997] L. Piegl and W. Tiller, The NURBS book, 2nd ed., Springer, 1997.

[Ramezani 2012] S. Ramezani, "A micro scale geometrically non-linear Timoshenko beam model based on strain gradient elasticity theory”, Int. J. Nonlin. Mech. 47:8 (2012), 863-873.

[Ramezani 2013] S. Ramezani, "Nonlinear vibration analysis of micro-plates based on strain gradient elasticity theory", Nonlinear Dynam. 73:3 (2013), 1399-1421.

[Reinaldo Goncalves et al. 2016] B. Reinaldo Goncalves, J. Jelovica, and J. Romanoff, "A homogenization method for geometric nonlinear analysis of sandwich structures with initial imperfections", Int. J. Solids Struct. 87 (2016), 194-205.

[Rickert et al. 2019] W. Rickert, E. N. Vilchevskaya, and W. H. Müller, "A note on Couette flow of micropolar fluids according to Eringen's theory”, Math. Mech. Complex Syst. 7:1 (2019), 25-50.

[Stölken and Evans 1998] J. S. Stölken and A. G. Evans, "A microbend test method for measuring the plasticity length scale", Acta. Mater. 46:14 (1998), 5109-5115.

[Thai et al. 2014] C. H. Thai, S. Kulasegaram, L. V. Tran, and H. Nguyen-Xuan, "Generalized shear deformation theory for functionally graded isotropic and sandwich plates based on isogeometric approach", Comput. Struct. 141 (2014), 94-112.

[Thai et al. 2017] H.-T. Thai, T. P. Vo, T.-K. Nguyen, and S.-E. Kim, “A review of continuum mechanics models for size-dependent analysis of beams and plates", Compos. Scruct. 177 (2017), 196-219.

[Timoshenko and Goodier 1970] S. P. Timoshenko and J. N. Goodier, Theory of elastic stability, 3rd ed., McGraw-Hill, New York, 1970.

[Tran and Kim 2018] L. V. Tran and S.-E. Kim, "Stability analysis of multi-layered plates subjected to partial edge compression with and without initial imperfection", Compos. Scruct. 205 (2018), 26-41. 
[Tran et al. 2013] L. V. Tran, A. J. M. Ferreira, and H. Nguyen-Xuan, "Isogeometric analysis of functionally graded plates using higher-order shear deformation theory", Compos. Part B Eng. 51 (2013), 368-383.

[Tran et al. 2015] L. V. Tran, J. Lee, H. Nguyen-Van, H. Nguyen-Xuan, and M. A. Wahaba, "Geometrically nonlinear isogeometric analysis of laminated composite plates based on higher-order shear deformation theory", Int. J. Nonlin. Mech. 72 (2015), 42-52.

[Turner and Wiehn 2001] J. A. Turner and J. S. Wiehn, "Sensitivity of flexural and torsional vibration modes of atomic force microscope cantilevers to surface stiffness variations", Nanotechnology 12:3 (2001), 322-330.

[Vo and Nanakorn 2020] D. Vo and P. Nanakorn, "Geometrically nonlinear multi-patch isogeometric analysis of planar curved Euler-Bernoulli beams", Comput. Methods Appl. Mech. Engrg. 366 (2020), art. id. 113078.

[Wang et al. 2010] B. Wang, J. Zhao, and S. Zhou, "A micro scale Timoshenko beam model based on strain gradient elasticity theory", Eur. J. Mech. A Solids 29:4 (2010), 591-599.

[Xia et al. 2010] W. Xia, L. Wang, and L. Yin, "Nonlinear non-classical microscale beams: static bending, postbuckling and free vibration”, Internat. J. Engrg. Sci. 48:12 (2010), 2044-2053.

[Yang et al. 2002] F. Yang, A. C. M. Chong, D. C. C. Lam, and P. Tong, "Couple stress based strain gradient theory for elasticity”, Int. J. Solids Struct. 39:10 (2002), 2731-2743.

Received 21 Nov 2019. Revised 18 May 2020. Accepted 23 Jun 2020.

LOC V. TRAN: tranvinhloc@tdtu.edu.vn

Faculty of Civil Engineering, Ton Duc Thang University, Ho Chi Minh City, Vietnam

JARKKO NIIRANEN: jarkko.niiranen@aalto.fi

Department of Civil Engineering, Aalto University, Espoo, Finland 


\section{MATHEMATICS AND MECHANICS OF COMPLEX SYSTEMS}

\author{
EDITORIAL BOARD \\ ANTONIO CARCATERRA \\ ERIC A. CARLEN \\ FRANCESCO DELL'ISOLA \\ RAFFAELE ESPOSITO \\ ALbERT FANNJIANG \\ GILLES A. FRANCFORT \\ Pierangelo Marcati \\ Peter A. Markowich \\ MARTIN OSTOJA-STARZEWSKI \\ PiERRE SEPPECHER \\ David J. STEIGMANN \\ Paul Steinmann \\ PIERRE M. SuQueT \\ MANAGING EDITORS \\ MICOL AMAR \\ EMILIO BARCHIESI \\ MARTIN OsTOJA-STARZEWSKI \\ HONORARY EDITORS \\ TEODOR ATANACKOVIĆ \\ VICTOR BERDICHEVSKY \\ GuY BouchitTÉ \\ FELIX DARVE \\ CARlo Marchioro \\ ERRICO PRESUTTI \\ MARIO PULVIRENTI \\ LuCIO Russo \\ ADVISORY BOARD \\ Holm ALTENBACH \\ HARM ASKES \\ ANDREA BRAIDES \\ MAURO CARFORA \\ ERIC DARVE \\ FABRIZIO DAVÌ \\ ANNA DE MASI \\ Emmanuele DiBenedetto \\ VICTOR A. EREMEYEV \\ BERNOLD FIEDLER \\ IRENE M. GAMBA \\ PIERRE GERMAIN \\ SERgey GaVRiLyuk \\ Timothy J. Healey \\ ROBERT P. LIPTON \\ ANGELO LUONGO \\ JUAN J. MANFREDI \\ JEAN-JACQUES MARIGO \\ ANIL MISRA \\ ROBERTO NATALINI \\ Thomas J. Pence \\ ANDREY PIATNITSKI \\ Miguel A. F. SANJUAN \\ A. P. S. Selvadurai \\ MirosLaV ŠILHAVÝ \\ GEORG STADLER \\ Guido SwEERS \\ LEV TRUSKINOVSKY \\ JUAN J. L. VELÁZQUEZ \\ VINCENZO VESPRI \\ VITALY VOLPERT
}

\section{msp.org/memocs}

Università di Roma "La Sapienza", Italia

Rutgers University, USA

(CO-CHAIR) Università di Roma "La Sapienza", Italia

(TREASURER) Università dell'Aquila, Italia

University of California at Davis, USA

(CO-CHAIR) Université Paris-Nord, France

Università dell'Aquila, Italy

DAMTP Cambridge, UK, and University of Vienna, Austria

(CHAIR MANAGING EDITOR) Univ. of Illinois at Urbana-Champaign, USA

Université du Sud Toulon-Var, France

University of California at Berkeley, USA

Universität Erlangen-Nürnberg, Germany

LMA CNRS Marseille, France

Università di Roma "La Sapienza", Italia

Università degli Studi dell'Aquila, Italy

(CHAIR MANAGING EDITOR) Univ. of Illinois at Urbana-Champaign, USA

University of Novi Sad, Serbia

Wayne State University, USA

Université du Sud Toulon-Var, France

Institut Polytechnique de Grenoble, France

Università di Roma "La Sapienza", Italia

Università di Roma Tor Vergata, Italy

Università di Roma "La Sapienza", Italia

Università di Roma "Tor Vergata", Italia

Otto-von-Guericke-Universität Magdeburg, Germany

University of Sheffield, UK

Università di Roma Tor Vergata, Italia

Università di Pavia, Italia

Stanford University, USA

Università Politecnica delle Marche, Ancona (I), Italy

Università dell'Aquila, Italia

Vanderbilt University, USA

Gdansk University of Technology, Poland

Freie Universität Berlin, Germany

University of Texas at Austin, USA

Courant Institute, New York University, USA

Université Aix-Marseille, France

Cornell University, USA

Louisiana State University, USA

Università dell'Aquila, Italia

University of Pittsburgh, USA

École Polytechnique, France

University of Kansas, USA

Istituto per le Applicazioni del Calcolo "M. Picone", Italy

Michigan State University, USA

Narvik University College, Norway, Russia

Universidad Rey Juan Carlos, Madrid, Spain

McGill University, Canada

Academy of Sciences of the Czech Republic

Courant Institute, New York University, United States

Universität zu Köln, Germany

École Polytechnique, France

Bonn University, Germany

Università di Firenze, Italia

CNRS \& Université Lyon 1, France Angelo Vulpiani \& Università di Roma La Sapienza, Italia

MEMOCS (ISSN 2325-3444 electronic, 2326-7186 printed) is a journal of the International Research Center for the Mathematics and Mechanics of Complex Systems at the Università dell'Aquila, Italy.

Cover image: "Tangle" by @ John Horigan; produced using the Context Free program (contextfreeart.org).

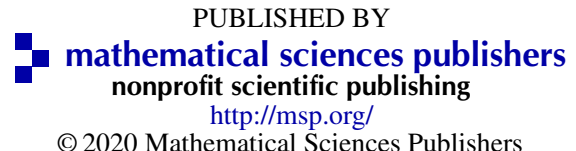

(C) 2020 Mathematical Sciences Publishers 
Mathematics and Mechanics of Complex Systems vol. 8 no. 4

On a stochastic approach to model the double phosphorylation/dephosphorylation cycle

Alberto Maria Bersani, Alessandro Borri, Francesco Carravetta,

Gabriella Mavelli and Pasquale Palumbo

A new comprehensive approach for bone remodeling under medium and high mechanical load based on cellular activity

Daniel George, Rachele Allena, Céline Bourzac, Stéphane Pallu, Morad Bensidhoum, Hugues Portier and Yves Rémond

Models for drug release of gentamicin in a polylactic acid matrix

Anna S. Morozova, Elena N. Vilchevskaya, Wolfgang H. Müller and Nikolay M. Bessonov

Analytical mechanics allows novel vistas on mathematical epidemic dynamics modeling

\section{Paul Steinmann}

A geometrically nonlinear Euler-Bernoulli beam model within strain gradient elasticity with isogeometric analysis and lattice structure applications

Loc V. Tran and Jarkko Niiranen

MEMOCS is a journal of the International Research Center for the Mathematics and Mechanics of Complex Systems at the Università dell' Aquila, Italy.

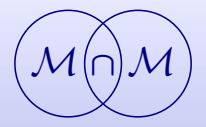

